\title{
Lumbago is different from prolapse of lumbar intervertebral disc
}

\author{
ZHOU Wei ${ }^{1}$, QIU Yan-bo ${ }^{1}$, ZHOU Shao-qiu ${ }^{1}$, ZHAO Wei ${ }^{2}$ \\ 1.Xuzhou City,Jiangsu Province,Juning County,Gao Zuozhen Caozhuang Community Health Service Station Xuzhou City,Jiangsu \\ Province, 221231, China
}

2.Community Health Service Station,Guancheng Town,Guanshan Town,Juning County,Xuzhou City,Jiangsu Province,China

Abstract:There are many errors found in the content of textbook in National Higher Education.For example:One of the errors found in the "Surgery"version no 418 is about movement system disease section.In chapter 677 Section III,the content misinterpretation of the cause of low back pain is the intervertebral disc protrusion that stimulate the outer annulus and the posterior longitudinal ligament in the sinus nerve fibers.For the past twenty years, feedbacks had been reflected repeatedly to the involved party but no one had admitted the contents of the textbook are wrong. The errors had brought great economy loss,physical and mental pain to patients.Every year,the country has to spend billions of dollars in the waste of medical reform reimbursement. This article is aimed to discuss about low back pain is not due to lumbar disc herniation.

Key words: Waist soft tissue anxious;Chronic injury;Anatomy and physiology;Lumbar disc herniation

\section{Introduction}

This study aims to prove that low back pain is not due to lumbar disc herniation.For many years,patients have been misdiagnosed.After careful study and analysis,it can be summed up that the problem is due to the wrong contents in the textbook.The"Surgery"textbook mentioned that low back pain is not a lumbar disc herniation.It is internationally recognized academic controversy(6).Imaging examination must be done in order to provide scientific reference for medical students.We carefully review the basic knowledge of anatomy and physiology with combined of clinical practice in order to correct the fact of low back pain is not due to lumbar disc herniation.

Copyright (C) 2017 ZHOU Wei, et.al.

doi: http://dx.doi.org/10.18686/aem.v6.78

This is an open-access article distributed under the terms of the Creative Commons Attribution Unported License

(http://creativecommons.org/licenses/by-nc/4.0/), which permits unrestricted use, distribution, and reproduction in any medium, provided the original work is properly cited. 


\section{Methods:Review human anatomy and physiology knowledge with}

\section{combined of clinical practice}

(1) The spine is the hard tissue of the movement system. The front of the spine is the vertebral body.The spine consists of 24 vertebrae,sacrum,coccyx and 23 discs. Each lumbar vertebrae and lumbar intervertebral has 5 discs.The intervertebral disc is a fibrous cartilage that connects two vertebral bodies.The lumbar disc is the cushion between the two lumbar vertebrae; the central part of the disc is a colloid called nucleus.Nucleus pulposus is around the fibrous ring.Peripheral fibrous rings are slightly raised in the opposite direction.Its shape is like the bottom of the two plates together,above the plate knock on the top of the vertebral body.The following plate knocked on the following vertebral body,so that the upper and lower vertebral body is not easy to slip. The function of lumbar intervertebral disc is to reduce the direct friction between the vertebral body,buffer,and load-bearing function.From the mechanical point of view,people in daily life with a variety of work in the posture, in addition to sleeping,side,lying life and work,vertebral body in the above tend to squeeze the following disc.Lumbar disc after several decades of extrusion,coupled with aging,and blood decay.Intervertebral disc has different degrees of injury,bulging,and prominent.So,every elderly have lumbar disc herniation. This is normal physiological changes of the elderly and it shows no symptoms of any disease.Clinical investigation had been performed in outpatient on the low back pain who have the symptoms of lumbar disc herniation patients.Imaging studies shows it is the lumbar disc herniation,but in fact only one of ten thousand of the following people has root sciatica.

(2) The muscles on the back of spine are the waist muscles.Ligament is the soft tissue of the movement system.Lumbar muscle is an important factor in maintaining normal waist stability and leaning forward.Lumbar muscle from the iliac crest to iliotibular ligament have up to 12 ribs;lumbar transverse process caused the spine occurred lateral flexion. There were 3 groups of lumbar muscle:1.Dorsal group.Shallow latissimus dorsi,middle erector spine,deep cleft muscle, and gyroscope erector spine are located between the spinous process and transverse process. They play a role inside the muscle contraction.It can make the lateral flexion and rotation.2.The front side group.Intra-abdominal oblique,abdominal oblique, and rectus abdominis are the flexor of the spine.3.Lateral group.Psoas muscle and lateral muscle.Psoas muscle starts from the chest 12 and lumbar vertebral body side,intervertebral disc and transverse process roots,downward extension and iliac muscle synthesis for the iliopsoas and stops at the femur minor rotor. There is a strengthening of the hip effect.If the lower limbs are fixed,that can make the spine before the bend.

(3) The editor of"Surgery"publisher does not admit the contents of the textbook is wrong

and replies that lumbar disc herniation of low back pain is an internationally recognized academic controversy category.Imaging examination must be done in order to provide scientific basis for medical students. This indicates that the textbook is copied from false papers,and intentionally input into the textbooks in China.

(4) Low back pain is common among young labor.Incorrect long posture can cause injury in the waist muscles, ligament,tense fatigue, leading to muscle spasms and pain.Sudden force to the waist,excessive muscle load or uncoordinated contraction will lead to muscle and ligament tear,bleeding and swelling.Damage occurred in the sacral spine muscle, and back fascia attachment.It can be seen in the interspinous, transverse intercostal, and sacroiliac ligament tear.Acute treatment is wrong and cannot completely cure the patients. The formation of chronic lumbar muscle injury is due to cold and tired.Climate change will aggravate the symptoms. The pains are from the muscles and ligaments,not from neuralgia.However,it is not disc herniation that stimulates the outer ring and the posterior longitudinal ligament in the sinus nerve fibers that caused muscle pain or neuralgia.During diagnosis,doctor performs hand touching to the waist of the affected area and senses obvious tenderness point.The pain point is not from neuralgia.Treatment of lumbar pain 
is by small needle therapy.Once cured,the pain point will not recurrence.There is no effect shows in lumbago with lumbar disc herniation approach treatment,it is only worsen the symptoms.

\section{Case:1.Lumbar spine ligament injury was misdiagnosed as lumbar disc herniation}

The patient name is Liu,male,55 years old,Han nationality.He is from River Town East Village,Hubin District,Suqian City,Jiangsu Province,China.In June 2010,the patient visited our health clinic.

Case description:

A year ago,patient had experience back pain and went to the hospital for treatment. The scan showed that it is due to lumbar disc herniation.Few local hospital scans also diagnosed as lumbar disc herniation.Same treatment had been applied including traction,physical therapy,drugs and other treatment.There is not much effect and patient still in pain,cannot perform work or self-care.Then,the patient decided to go to a hospital in Nanjing for treatment.The hospital scan showed that it is lumbar disc herniation and the patient had decided to do lumbar intervertebral disc surgery.After all medical cost has been paid,patient's eldest daughter is worried about the risk or any side effects of the surgery.Then,they cancelled the surgery and sneak out from the hospital.

Diagnosis:The patient is diagnosed with the touch pressure,lumbar spine 3,4 between the tenderness point,waist 5,sacral 1 interspinous tenderness point; bilateral iliac crest later,about $5 \mathrm{~cm}$ long tenderness.

Results of diagnosis:Lumbar spine ligament injury.Treatment:Perform small needle treatment.Patient had recovered after one treatment.After half a month,patient's neighbors came for treatment and mentioned that Liu had not been working more than a year,but after the treatment,he can perform his carpenter work now.

Over the past year,lots of hospital's physicians that diagnose according to teaching materials on the low back pain as lumbar intervertebral protrusion.Even using imaging science to do scientific basis,the disease was still misdiagnosed.Exacerbations, resulting in great physical and mental pain while causing significant losses economically.Lumbar disc in the first half of the spine,low back pain is caused by spinal muscular injury.It does not need to use imaging to do scientific basis,palpation can be diagnosed by hand,and it can be cured with a small needle therapy.It proves that low back pain is not due to lumbar disc herniation.

\section{Case:2.Acute soft tissue injury was misdiagnosed as lumbar disc}

\section{herniation}

A female patient named Song Ti-Qing,71 year old,Han nationality,living in Cao Village,Suining County,Xuzhou City,Jiangsu Province,China.On March 22,2017,patient's husband came to ask for a doctor visit to his house as his wife had injured her waist when fell down twenty days ago.She was hospitalizing in the county people's hospital.The imaging examination showed that it is lumbar disc herniation.Then,she went to the city hospital but was rejected for surgery due to her prolongs sickness. She then returned to the county people's hospital.Patient claims prolonged pains and cried.She was given oral tramadol.The treatment is ineffective because once the drug is over,the pains continue and she continues shouting in pain. The drug had cost her more than 6000 Yuan.The county people's hospital had no other better options and recommended the patient to go home to do small needle treatment.

Diagnosis:The patient claimed pain. When touch the waist by hand,large area are tender.The diagnosis showed acute soft tissue loss of the waist. 
Treatment:Eight-character therapy plus low back acupressure.After the treatment,patients felt comfortable waist and $80 \%$ of the problem cured.

In this case,patient's pain is due to the injury of waist soft tissue,but doctors misdiagnosed the low back pain as lumbar disc herniation. This shows that even imaging can still lead to misdiagnose.Chinese treatment and acupuncture is an effective method to cure.It is also shows that low back pain is not due to lumbar disc herniation.

\section{Conclusion}

In summary,on the back of the lumbar spine is the muscles and the lumbar disc are in the first half of the spine;and pain is due to injury in the waist chronic soft tissue adhesions. This is an undisputed fact to proof that low back pain is not the symptoms of lumbar disc herniation.Imaging studies are not necessary in diagnosis.Hand touch on the affected area with significant tenderness will proof the symptom. One time treatment with a small needle therapy to release the adhesion of the tissue will cure the pain point with no recurrence.

\section{References}

1. Yang Zhanhui,Sun Jianhua,Ding Hao.Lumbar disc herniation of the efficacy of the evaluation criteria[J].Journal of cervical and low back pain.1999(01)

2. Fairbank JC,Nan Deng Kun.Low back pain score table[J].Foreign Medicine(Physical Medicine and Sports Medicine).1981(02)

3. Dang Guoan,Li Tianzhu,Johnson,Hou Dongfang.Effect observation of lumbar disc herniation systematic conservative treatment program[A].Chinese Society of acupuncture and moxibustion minimally invasive knife professional committee of the second academic seminar academic papers[C].2010 